Vol. 50 (1994) [435-443]

\title{
USE OF THE RABINOWITSCH POLYNOMIAL TO DETERMINE THE CLASS GROUPS OF A REAL QUADRATIC FIELD
}

\author{
R.A. MOLLIN
}

The main result is a necessary and sufficient condition for the class group of a real quadratic field to be determined by primality properties of the well-known Rabinowitsch polynomial.

\section{Notation and Preliminaries}

Throughout $d$ will be a positive, square-free integer and $\omega=(\sigma-1+\sqrt{d}) / \sigma$ where

$$
\sigma=\left\{\begin{array}{ll}
2 & \text { when } d \equiv 1(\bmod 4) \\
1 & \text { otherwise }
\end{array} .\right.
$$

The discriminant $\Delta$ of $K=Q(\sqrt{d})$ is given by $\Delta=(2 / \sigma)^{2} d$. If $[\alpha, \beta]$ denotes the module $\{\alpha x+\beta y: x, y \in Z\}$, then the maximal order $O_{\Delta}$ of $\mathrm{K}$ is given by $O_{K}=[1, \omega]$. For $\alpha \in K$, we use $\bar{\alpha}$ to denote the algebraic conjugate of $\alpha$ and $N(\alpha)$ to denote the value of $\alpha \bar{\alpha}$, the norm of $\alpha$.

An ideal of $O_{\Delta}$ can be written as $I=[a, b+c \omega]$ where $a, b, c \in \mathbf{Z}$ with $a, c>0$, $c|b, c| a$, and $a c \mid N(b+c \omega)$. Conversely, if $a, b, c \in \mathbf{Z}$ with $c|b, c| a$, and $a c \mid N(b+c \omega)$ then $[a, b+c \omega]$ is an ideal of $O_{K}$. For an ideal $I=[a, b+c \omega]$ with $a, c>0$ the norm of $I, N(I)$, is given by $N(I)=a c>0$. If $c=1$, then $I$ is said to be a primitive ideal. The conjugate of $I=[a, b+\omega]$ is $I^{\prime}=[a, b+\bar{\omega}]$. A primitive ideal $I$ is reduced if it does not contain any non-zero element $\alpha$ such that both $|\alpha|<N(I)$ and $|\bar{\alpha}|<N(I)$.

At this juncture, we introduce the connection between reduced ideals and continued fractions. Let $\alpha \in K$, then we can write $\alpha=\left(P_{0}+\sqrt{d}\right) / Q_{0}$ where $P_{0}, Q_{0} \in \mathbb{Z}$. If we put $a_{0}=\lfloor\alpha\rfloor$ (where \lfloor\rfloor is the greatest integer function) and define

$$
\begin{aligned}
P_{i+1} & =a_{i} Q_{i}-P_{i} \\
Q_{i} Q_{i+1} & =d-P_{i+1}^{2} \\
a_{i+1} & =\left\lfloor\left(P_{i+1}+\sqrt{d}\right) / Q_{i+1}\right\rfloor \quad(i=0,1,2, \ldots)
\end{aligned}
$$

Received 19 January 1994

The author's research is supported by NSERC Canada grant \# A8484.

Copyright Clearance Centre, Inc. Serial-fee code: 0004-9729/94 SA2.00+0.00. 
then

$$
\alpha=\left\langle a_{0}, a_{1}, a_{2}, \ldots, a_{i}, \ldots\right\rangle
$$

is the continued fraction expansion of $\alpha$. Moreover,

THEOREM 1.1. Let $I_{1}=I=[a, b+\omega]$ be a reduced ideal of $O_{\Delta}$. If $\alpha=$ $(b+\omega) / a$ then all of the reduced ideals in the same equivalence class as $I$ and only these are given by

$$
I_{j}=\left[Q_{j-1} / \sigma,\left(P_{j-1}+\sqrt{d}\right) / \sigma\right]
$$

for $j=1,2,3, \ldots$ where the values of the $P_{j}$ 's and $Q_{j}$ 's are found by expanding $\alpha$ into a continued fraction.

Furthermore,

THEOREM 1.2. If $I$ is a reduced ideal of $O_{\Delta}$ then $N(I)<\sqrt{\Delta}$. If $I$ is a primitive ideal of $O_{\Delta}$ such that $N(I)<\sqrt{\Delta} / 2$ then $I$ is a reduced ideal of $O_{\Delta}$.

By Theorem 1.2, then, there can only be a finite number of reduced ideals of $O_{\Delta}$ and since all the $I_{j}$ 's from Theorem 1.1 are reduced then we see that the sequence of reduced ideals $I_{1}, I_{2}, I_{3}, \ldots, I_{j}, \ldots$ produced by the continued fraction expansion must be purely periodic; that is, there must exist a minimal positive $l \in \mathbf{Z}$ such that $I_{l+1}=I_{1}$. We call $l(I)=l$ the period length of the continued fraction expansion of $\alpha$.

We let $C_{\Delta}$ denote the class group of $K$ and $h_{\Delta}$ its order; that is, the class number of $K$. Equivalence of ideals is denoted by $I \sim J$. Let $\{I\}$ denote the class of $I$ in $C_{\Delta}$. We also have,

THEOREM 1.3. (1) If $I$ is a reduced ideal of $O_{\Delta}$ then there exists an ideal of $J \sim I$ such that $N(J)<\sqrt{\Delta} / 2$.

(2) $C_{\Delta}$ is generated by the primitive ideals $I$ with $N(I)<\sqrt{\Delta} / 2$.

Immediate from the above is,

Theorem 1.4. Let $\Delta>0$ be a discriminant and $J_{1}, J_{2}, \ldots, J_{k}$ primitive ideals, then $C_{\Delta}=\bigcup_{i=1}^{k}\left\{I_{i}\right\}$ if and only if for each prime $p<\sqrt{\Delta} / 2$ which is noninert, there exists an integer $i$ with $1 \leqslant i \leqslant k$ and a reduced ideal $I_{i}=\left[a_{i}, b_{i}+\omega\right] \sim J_{i}$ such that in the continued fraction expansion of $\left(b_{i}+\omega\right) / a_{i}$ we have $Q_{j} / \sigma=p$ for some $j$ with $1 \leqslant j \leqslant l_{i}=l\left(I_{i}\right)$.

Now we define the Rabinowitsch polynomial which we wish to bring into the picture.

DEFINITION 1.1. The Rabinowitsch polynomial for $K$ is

$$
f_{\Delta}(x)=-x^{2}+(\sigma-1) x+(\Delta-\sigma+1) / 4 .
$$

In $[4$, Lemma 3.1, p.830] we proved the following useful result. 
Lemma 1.1. If $p<\sqrt{\Delta} / 2$ is prime then $f_{\Delta}(x) \equiv 0(\bmod p)$ for some integer $x$ with $1 \leqslant x \leqslant\lfloor\omega\rfloor$ if and only if $p$ is not inert in $K$ (that is, all non-inert primes $p<\sqrt{\Delta} / 2$ divide $f_{\Delta}(x)$ for some integer $x$ with $1 \leqslant x \leqslant\lfloor\omega\rfloor$ and they are the only such prime divisors of $f_{\Delta}(x)$ less then $\left.\sqrt{\Delta} / 2\right)$.

Thus from Lemma 1.1 and Theorem 1.4, we have the well-known class number 1 criterion (for example see Hendy [2]), which seems to have been rediscovered by Louboutin [3].

COROLLARY 1.1. If $\Delta>0$ is a discriminant then $h_{\Delta}=1$ if and only if $p=$ $Q_{i} / \sigma$ for some $i$ with $0<i<l$ in the continued fraction expansion of $\omega$, for all primes $p<\sqrt{\Delta} / 2$ which divide $f_{\Delta}(x)$ for any $x$ with $1 \leqslant x \leqslant \sqrt{\Delta} / 2$.

We shall also have need of the following concept introduced in [7].

DEFINITION 1.2. Let $\Delta>0$ be a discriminant and let $I=[a,(b+\sqrt{\Delta}) / 2]$ be a reduced ideal in $O_{\Delta}$. If $I$ is in an ambiguous class (that is, $I \sim I^{\prime}$ ) then in the continued fraction expansion of $(b+\sqrt{\Delta}) / 2 a$ we must have $I^{\prime}=I_{p}$ for some integer $p$ with $1 \leqslant p \leqslant l(I)$. We call $p=p(I)$ the palindromic index of $I$.

In [7] we proved,

THEOREM 1.5. Let $\Delta>0$ be a discriminant and let $I=[a,(b+\sqrt{\Delta}) / 2]$ be in a reduced ambiguous class of $C_{\Delta}$. Let $Q_{i}$ be in the continued fraction expansion on $(\sqrt{\Delta}+b) / 2 a$. Then $Q_{i} / \sigma$ is a square-free divisor of $\Delta$ if and only if one of the following holds:

(a) $p=l$ and $i=0$ or $l$

(b) $p$ is even and $i=p / 2$

(c) $p$ and $l$ have the same parity and $i=(p+l) / 2$.

The next result which we proved in [6] is the key to our Rabinowitsch criterion for determination of $C_{\Delta}$.

THEOREM 1.6. Let $\Delta>0$ be a discriminant and let $I=[a, b+\omega]$ be a reduced ideal in $O_{\Delta}$. If, in the continued fraction expansion of $(b+\omega) / a, Q_{i} / \sigma$ is a prime for some positive integer $i \leqslant l(I)=l$, then $f_{\Delta}(x) \equiv 0\left(\bmod Q_{i} / \sigma\right)$ for exactly $a_{i}+1$ values of $x$ with $1 \leqslant x \leqslant\lfloor\omega\rfloor$ whenever $Q_{i} / \sigma$ is not a divisor of $\Delta$. If $Q_{i} / \sigma$ is either 1 or a prime divisor of $\Delta$ for $0<i \leqslant l$ then $i=l=p$ or $i=p / 2$ or $i=(p+l) / 2$ and there are exactly $\left\lfloor\left(a_{i}+1\right) / 2\right\rfloor$ such values of $x$. If $Q_{i} / \sigma>1$ is not prime then there are at least that many values of $x$.

The reader is referred to [8] for further details and proofs of the above results. 


\section{THE CRITERION}

First we need some definitions.

DEFINITION 2.1. If $\Delta>0$ is a discriminant and $I=[a,(b+\sqrt{\Delta}) / 2]$ is a reduced ideal in $O_{\Delta}$ then in the following we are considering the continued fraction expansion of $(b+\Delta) / 2 a$. Let

$T(I)=\left\{j: 1 \leqslant j \leqslant l(I)=l\right.$ with $Q_{j} / \sigma<\sqrt{\Delta} / 2$ and $Q_{j} / \sigma$ a prime counted once, that is, without multiplicity $\}$ and,

$R(I)=\{j: 1 \leqslant j \leqslant l$ and one of $j=l=p(I)=p$ or $j=p / 2$ or $j=(p+l) / 2$ holds $\}$.

DEFINITION 2.2. If $\Delta>0$ is a discriminant and $p$ is a given prime then set

$$
S_{f}(p)=\mid\left\{x: 1 \leqslant x \leqslant\left\lfloor\omega_{\Delta}\right\rfloor \text { with } p \mid f_{\Delta}(x)\right\} \mid .
$$

REMARK 2.1. We observe that $R(I)=\emptyset$ unless $I$ is in an ambiguous class. Moreover, we need only concern ourselves about multiplicity in $T(I)$ when $I$ is in an ambiguous class, (see Theorem 1.5).

Now we are in a position to prove the main result.

2.1. (The Criterion). Let $\Delta>0$ be a discriminant. Then $C_{\Delta}=\bigcup_{i=1}^{k}\left\{J_{i}\right\}$ for primitive ideals $J_{i}$ if and only if there exists a reduced ideal $I_{i} \sim J_{i}$ for each $i$ with $1 \leqslant i \leqslant k$ such that

$$
\sum_{p<\sqrt{\Delta} / 2} S_{f}(p)=\sum_{i=1}^{k} \sum_{j \in T_{i}-R_{i}}\left(a_{(i, j)}+1\right)
$$

where the left hand sum ranges over unramified primes, and

$$
\sum_{p<\sqrt{\Delta} / 2} S_{f}(p)=\sum_{i=1}^{k} \sum_{j \in R_{i} \cap T_{i}}\left\lfloor\left(a_{(i, j)}+1\right) / 2\right\rfloor
$$

where the left hand sum ranges over ramified primes and where $T_{i}=T\left(I_{i}\right), R_{i}=R\left(I_{i}\right)$, and $a_{(i, j)}=\left\lfloor\left(P_{j}+\sqrt{D}\right) / Q_{j}\right\rfloor$ in the continued fraction expansion of $I_{i}$.

Proof: By Theorem 1.6, the right hand sides of (1)-(2) actually represent $\sum S_{f}(p)$ where the sum ranges over only those $p<\sqrt{\Delta} / 2$ which appear as some $Q_{(i, j)} / \sigma$ in the continued fraction expansion of $\left(b_{i}+\omega\right) / a_{i}$ where $I_{i}=\left[a_{i}, b_{i}+\omega\right]$. Therefore, the equality fails to hold precisely when some prime $p<\sqrt{\Delta} / 2$ does not appear on the right but does (as it must by Lemma 1.1) appear on the left. The result now follows from Theorem 1.1-1.5. 
The following illustrates Theorem 2.1.

EXAMPLE 2.1: Let $\Delta=2^{3} \cdot 11 \cdot 23=2024$. Then $f_{\Delta}(x)=506-x^{2}$ and we have,

\begin{tabular}{l|ll|l}
$\boldsymbol{x}$ & $f_{\Delta}(x)$ & & \\
\hline $\mathbf{1}$ & $5 \cdot 101$ & $x$ & $f_{\Delta}(x)$ \\
2 & $2 \cdot 251$ & 12 & $2 \cdot 181$ \\
3 & $7 \cdot 71$ & 13 & 337 \\
4 & $2 \cdot 57^{2}$ & 14 & $2 \cdot 5 \cdot 31$ \\
5 & $13 \cdot 37$ & 15 & 281 \\
6 & $2 \cdot 5 \cdot 47$ & 16 & $2 \cdot 5^{3}$ \\
7 & 457 & 17 & $7 \cdot 31$ \\
8 & $2 \cdot 13 \cdot 17$ & 18 & $2 \cdot 7 \cdot 13$ \\
9 & $5^{2} \cdot 17$ & 19 & $5 \cdot 29$ \\
10 & $2 \cdot 7 \cdot 29$ & 20 & $2 \cdot 53$ \\
11 & $5 \cdot 7 \cdot 11$ & 21 & $5 \cdot 13$ \\
& & 22 & $2 \cdot 11$
\end{tabular}

Thus, observing that the non-inert primes less than $\sqrt{\Delta} / 2$ are $2,5,7,13$, and 17 we have $S_{f}(2)=11, S_{f}(5)=9, S_{f}(7)=6, S_{f}(11)=2, S_{f}(13)=4$, and $S_{f}(17)=2$.

Now consider the ideals $I_{1}=[1, \omega], I_{2}=[2, \sqrt{506}], I_{3}=[5,1+\sqrt{506}]$, and $I_{4}=\{7,17+\sqrt{506}\}$.

The continued fraction expansion of $\omega$ is

$\begin{array}{cccc}i & 0 & 1 & 2 \\ P_{i} & 0 & 22 & 22 \\ Q_{i} & 1 & 22 & 1 \\ a_{i} & 22 & 2 & 22\end{array}$

The continued fraction expansion of $\sqrt{506} / 2$ is

$\begin{array}{cccc}i & 0 & 1 & 2 \\ P_{i} & 0 & 22 & 22 \\ Q_{i} & 2 & 11 & 2 \\ a_{i} & 11 & 4 & 22\end{array}$

The continued fraction expansion of $(1+\sqrt{506}) / 5$ is

$\begin{array}{cccccc}i & 0 & 1 & 2 & 3 & 4 \\ P_{i} & 1 & 19 & 10 & 18 & 21 \\ Q_{i} & 5 & 29 & 14 & 13 & 5 \\ a_{i} & 4 & 1 & 2 & 3 & 8\end{array}$


and, the continued fraction expansion of $(17+\sqrt{506}) / 7$ is

$\begin{array}{cccccccc}i & 0 & 1 & 2 & 3 & 4 & 5 & 6 \\ P_{i} & 17 & 18 & 8 & 9 & 16 & 14 & 17 \\ Q_{i} & 7 & 26 & 17 & 25 & 10 & 31 & 7 \\ a_{i} & 5 & 1 & 1 & 1 & 3 & 1 & 5 .\end{array}$

Hence, we have that $T_{1}=\emptyset, T_{2}=\{1,2\}, T_{3}=\{3,4\}$ and $T_{4}=\{2,6\}$. Also, $R_{1}=\{1,2\}=R_{2}$ whereas $R_{3}=\emptyset=R_{4}$. Therefore,

$$
\sum_{i=1}^{4} \sum_{j \in T_{i}-R_{i}}\left(a_{(i, j)}+1\right)=\sum_{i=3}^{4} \sum_{j \in T_{i}}\left(a_{(i, j)}+1\right)=4+9+2+6=21
$$

and

$$
\sum_{i=1}^{4} \sum_{j \in R_{i} \cap T_{i}}\left\lfloor\left(a_{(i, j)}+1\right) / 2\right\rfloor=\sum_{j \in T_{2}}\left\lfloor\left(a_{(2, j)}+1\right) / 2\right\rfloor=2+11=13 .
$$

On the other hand, for unramified $p$, we have

$$
\sum_{p<\sqrt{\Delta} / 2} S_{f}(p)=S_{f}(5)+S_{f}(7)+S_{f}(13)+S_{f}(17)=9+6+4+2=21
$$

and for ramified $p$ we have

$$
\sum_{p<\sqrt{\Delta} / 2} S_{f}(p)=S_{f}(2)+S_{f}(11)=11+2=13
$$

Hence, by Theorem 2.11 we have the $C_{\Delta}=\bigcup_{i=1}^{4}\left\{I_{i}\right\}$. Observe that $I_{3}^{2}=[25,9+\sqrt{506}]$ is not reduced but $I_{3}^{2} \sim I_{4}$ and in fact $I_{3}^{3} \sim I_{2}$. Therefore, $C_{\Delta}=\left\langle\left\{I_{3}\right\}\right\rangle$ with $h_{\Delta}=6$. Also, $C_{\Delta}=\left\langle\left\{I_{2}\right\}\right\rangle \times\left\langle\left\{I_{4}\right\}\right\rangle$ since $I_{2}^{2} \sim 1$ and $I_{4}^{3} \sim 1$.

Now we state a class number one criterion which is immediate from Theorem 2.1. In what follows we are considering only the continued fraction expansion of $\boldsymbol{\omega}$.

Corollary 2.1. Let $\Delta>0$ be a discriminant. Then $h_{\Delta}=1$ if and only if

$$
\sum_{p<\sqrt{\Delta} / 2} S_{f}(p)=\sum_{j=1}^{\lfloor l / 2\rfloor} \theta_{j}
$$

where

$$
\theta_{j}= \begin{cases}a_{j}+1 & \text { if } j \neq l / 2 \text { and } Q_{j} / \sigma \text { is prime } \\ \left\lfloor\left(a_{j}+1\right) / 2\right\rfloor & \text { if } j=l / 2 \text { and } Q_{j} / \sigma \text { is prime } \\ 0 & \text { otherwise. }\end{cases}
$$


REMARK 2.2. Corollary 2.1 is a much simpler criterion than that given by Lu [5] (see Theorem 2.2). Furthermore, Corollary 1.1 is an immediate consequence 2.1 in view of Lemma 1.1.

We now illustrate the ease of use of Corollary 2.1 .

EXAMPLE 2.2: Let $\Delta=4 \cdot 94$ then the continued fraction expansion of $\omega$ is

$\begin{array}{cccccccccccccccccc}i & 0 & 1 & 2 & 3 & 4 & 5 & 6 & 7 & 8 & 9 & 10 & 11 & 12 & 13 & 14 & 15 & 16 \\ P_{i} & 0 & 9 & 4 & 8 & 7 & 2 & 8 & 7 & 8 & 8 & 7 & 8 & 2 & 7 & 8 & 4 & 9 \\ Q_{i} & 1 & 13 & 6 & 5 & 9 & 10 & 3 & 15 & 2 & 15 & 3 & 10 & 9 & 5 & 6 & 13 & 1 \\ a_{i} & 9 & 1 & 2 & 3 & 1 & 1 & 5 & 1 & 8 & 1 & 5 & 1 & 1 & 3 & 2 & 1 & 18\end{array}$

Moreover,

\begin{tabular}{l|l}
$x$ & $f_{\Delta}(x)=94-x^{2}$ \\
\hline 1 & $3 \cdot 31$ \\
2 & $2 \cdot 3^{2} \cdot 5$ \\
3 & $5 \cdot 17$ \\
4 & $2 \cdot 3 \cdot 13$ \\
5 & $3 \cdot 23$ \\
6 & $2 \cdot 29$ \\
7 & $3^{2} \cdot 5$ \\
8 & $2 \cdot 3 \cdot 5$ \\
$9=\left\lfloor\omega_{\Delta}\right\rfloor$ & 13
\end{tabular}

The non-inert primes less than $\sqrt{\Delta} / 2$ are $2,3,5$, and 13. Also,

$$
S_{f}(2)+S_{f}(3)+S_{f}(5)+S_{f}(13)=4+6+4+2=16
$$

whereas,

$$
\sum_{j=1}^{8} \theta_{j}=2+4+6+4=16
$$

Hence, by Corollary $2.1, h_{\Delta}=1$.

REMARK 2.3. Lu's result [5] for $h_{\Delta}=1$ is stated in a rather complicated form involving solutions to diophantine equations as follows. We present it here since we wish to compare it now to our chief result.

Theorem 2.2. (Lu [5]) Let $\Delta>0$ be a discriminant. Then $h_{\Delta}=1$ if and only if $c+\sum_{i=1}^{l} a_{i}=\lambda_{1}(\Delta)+\lambda_{2}(\Delta)$ where $\lambda_{1}(\Delta)$ is the number of solutions of $x^{2}+4 y z=\Delta$ with integers $x, y, z \geqslant 0, \lambda_{2}(\Delta)$ is the number of solutions of $x^{2}+4 y^{2}=\Delta$ with 
integers $x, y \geqslant 0$ and

$$
c= \begin{cases}0 & \text { if } l \text { is even, } a_{l / 2} \text { is odd when } \Delta \equiv 1(\bmod 4) \\ 1 & \text { otherwise when } \Delta \equiv 1(\bmod 4) \\ 1 & \text { if } l \text { is even, } a_{l / 2} \text { is odd when } \Delta \equiv 0(\bmod 4) \\ 2 & \text { otherwise when } \Delta \equiv 0(\bmod 4)\end{cases}
$$

ReMARK 2.4. As noted by Dubois and Levesque in [1], Lu's $c+\sum_{i=1}^{l} a_{i}$ is precisely the number of all those ideals $[Q,(P+\sqrt{\Delta}) / 2]$ such that $P^{2}+4 Q Q^{\prime}=\Delta$ where $Q$ appears as some $Q_{i}$ in the continued fraction expansion of $\omega$. In Corollary 2.1 our $\sum_{j=1}^{\lfloor l / 2\rfloor} \theta_{j}$ is precisely the number of ideals (counted without multiplicity) $[Q,(P+\sqrt{\Delta}) / 2]$ where $P^{2}+4 Q Q^{\prime}=\Delta$ with $Q$ appearing as some prime $Q_{i}<\sqrt{\Delta} / 2$ in the continued fraction expansion of $\omega$. Thus, our result is easier to implement and closer to the kernel of truth of the matter in view of Theorem 1.3. For instance, if we look back to Example 2.2, and consider $I=[5,(P+\sqrt{\Delta}) / 2]$ we get that $P^{2}+4 \cdot 5 \cdot Q^{\prime}=\Delta$ precisely where $2 x=P=4,5,14,16$. Thus, there are exactly 4 ideals of type $I:[5,2+\sqrt{94}]=[5,7+\sqrt{94}] \sim[5,8+\sqrt{94}]=[5,3+\sqrt{94}]$. Moreover, these account for the exact four.values of $x: 2,3,7$, and 8 for which 5 divides $f_{\Delta}(x)$ when $x \leqslant\lfloor\omega\rfloor$; that is, $S_{f}(5)=4=a_{3}+1$. This is a rather neat illustration of how the phenomenom works.

In any case, the fact, missed by Dubois-Levesque [1] is that Lu's result is intimately linked to the divisor function $\tau(x)$ by its very nature, (here $\tau(x)$ denotes the number of positive divisors of $x$ ). All we have to do is recognise that $\lambda_{1}(\Delta)+\lambda_{2}(\Delta)$ is just $\sum_{P_{0} \leqslant x \leqslant\lfloor\omega\rfloor} \tau\left(f_{\Delta}(x)\right)$. To see this we observe that $x_{1}^{2}+4 y z=\Delta$ implies that $y z=$ $\left(\Delta-x_{1}^{2}\right) / 4=f_{\Delta}(x)$ where $x_{1}=\sigma x-\sigma+1$, and $x_{1}^{2}+4 y^{2}=\Delta$ is just $x_{1}^{2}+4 y z=\Delta$ with $y=z$. Finally, observe that since $y$ and $z$ are non-negative then $P_{0} \leqslant x \leqslant\lfloor\omega\rfloor$. Hence,

THEOREM 2.3. (Lu reinterpreted) [5] If $\Delta>0$ is a discriminant then

$$
\sum_{P_{0} \leqslant x \leqslant\lfloor\omega\rfloor} \tau\left(f_{\Delta}(x)\right)=\sum_{i=1}^{l} a_{i}+c
$$

where $c$ is as in Theorem 2.2.

If we now re-examine Example 2.2, we see that

$$
\sum_{i=1}^{l} a_{i}+c=\sum_{i=1}^{16} a_{i}+2=56
$$


whereas,

$$
\sum_{P_{0} \leqslant x \leqslant\lfloor\omega\rfloor} \tau\left(f_{\Delta}(x)\right)=56
$$

Hence, by Theorem 2.3, $h_{\Delta}=1$.

REMARK 2.5. In Lu's main result [5, Theorem 2, p.119] restated (in a different and better format) as Theorem 2.3, there is a case value of (what he calls) $\theta$ which is vacuous, when $\Delta \equiv 1(\bmod 4), l$ is even and $a_{l / 2}$ even. This case cannot occur because $a_{l / 2} Q_{l / 2}-P_{l / 2}=P_{l / 2+1}=P_{l / 2}$ implies that $P_{l / 2}=\left(a_{l / 2} / 2\right) Q_{l / 2}$ is even. However, $Q_{l / 2}$ is even and $D=P_{l / 2}^{2}+Q_{l / 2} Q_{l / 2-1}$ forcing $D$ to be even, a contradiction.

In conclusion, Theorem 2.1 is a completely general criterion for the determination of the class group of a real quadratic field, given in terms of the Rabinowitsch polynomial.

\section{REFERENCES}

[1] E. Dubois and C. Levesque, 'On determining certain real quadratic fields with class number one and relating this property to continued fractions and primality properties', Nagoya Math. J. 124 (1991), 157-180.

[2] M.D. Hendy, 'Applications of a continued fraction algorithm to some class number problems', Math. Comp. 28 (1974), 267-277.

[3] S. Louboutin, 'Continued fractions and real quadratic fields', J. Number Theory 30 (1988), 167-176.

[5] S. Louboutin, R.A. Mollin and H.C. Williams, 'Class numbers of real quadratic fields, continued fractions, reduced ideals, prime-producing quadratic polynomials, and quadratic residue covers', Canad. J. Math. 44 (1992), 824-842.

[5] H. Lu, 'On the class number of real quadratic fields', Sci-Sinica (special issue (II)) (1979), 118-130.

[6] R.A. Mollin, 'An efficient method for the determination of certain real quadratic fields of class number one', Utilitas Math. 40 (1991), 27-32.

[7] R.A. Mollin, 'The palindromic index - a measure of ambiguous classes with no ambiguous ideals in class groups of real quadratic orders', Sém. Théor. Nombres Bordeaux (to appear).

[8] H.C. Williams and M.C. Wunderlich, 'On the parallel generation of the residues for the continued fraction factoring algorithm', Math. Comp. 177 (1987), 405-423.

\footnotetext{
Department of Mathematics and Statistics

University of Calgary

Calgary, Alberta T2N 1N4

Canada
} 